\title{
Chemosensors and chemoreception
}

\author{
Jong-Heun Lee $\cdot$ Hyung Gi Byun
}

Published online: 28 February 2014

(C) Springer-Verlag Berlin Heidelberg 2014

The objective of this topical collection is to report on the most recent research into and applications of chemical sensing and olfaction. It focuses in particular on chemosensors (gas sensors) and chemoreception (artificial olfaction), and the interactions between them. With the widespread use of chemicalsensor devices, there has been much investigation of highly sensitive, selective, and stable chemoresistors using oxide semiconductors, conducting polymers, graphenes, and carbon nanotubes. The diversity of sensing materials provides a variety of benefits, including flexible design, low temperature (power) operation, and integration into portable devices, in addition to crucial " $3 \mathrm{~S}$ " requirements (sensitivity, selectivity, and stability), which will enable innumerable new applications using chemosensors. To realize these benefits, understanding and investigation of the mechanisms and materials of chemosensors are essential.

The information gathered from chemoresistors is extremely useful for enabling chemoreception in artificial olfaction devices. These devices, designed to mimic the mammalian sensory system, are gaining favor for odor and/or gas assessment applications in a variety of fields: environmental quality monitoring, food and beverage quality control, medical diagnosis, and others. In this paper collection, we invited front-line researchers to submit original research articles and critical reviews on chemosensors and chemoreception.

Published in the topical collection Chemosensors and Chemoreception with guest editors Jong-Heun Lee and Hyung-Gi Byun.

\section{J.-H. Lee $(\square)$}

Department of Materials Science and Engineering, Korea University,

Seoul 136-713, Republic of Korea

e-mail: jongheun@korea.ac.kr

H. G. Byun $(\square)$

Division of Electronics, Information and Communication

Engineering, Kangwon National University (Samcheok),

Kangwon-Do 245-711, Republic of Korea

e-mail: byun@kangwon.ac.kr
Altogether, this collection of papers should be a valuable source of information for all readers with an interest in chemosensors and chemoreception or related fields. We thank all authors for submitting their interesting contributions for this issue, the referees for their critical but constructive comments, and the Editorial Office and the Editors for their friendly cooperation.

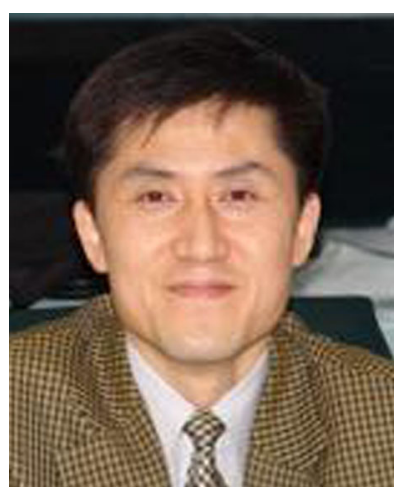

Jong-Heun Lee is a professor at the Department of Materials Science of Engineering, Korea University, Seoul, Korea. He received a PhD. in Materials Science and Engineering from Seoul National University, Korea. He has published over 200 original papers and four invited reviews. His main subjects are chemical sensors using oxide nanostructures and solid electrolytes and their functional applications. He is a junior member of the Korean Academy of Science and Technology.

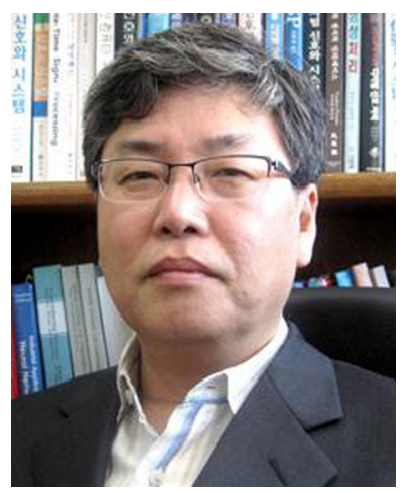

Hyung-Gi Byun is a professor at the Division of Electronics, Information and Communication Engineering Kangwon National University (Samcheok Campus), Korea. He received a $\mathrm{PhD}$. in Instrumentation and Analytical Science from the University of Manchester, UK. His research interests are pattern recognition and signal processing for intelligent sensing systems and its medical and industrial applications. He has been involved in more than 30 different research projects domestically and internationally. Dr Byun has authored approximately 80 publications and served as a committee member for ISOCS (International Society for Olfaction and Chemical Sensing) and the Korea Sensor Society. 